\title{
On the Reynolds number scaling of vorticity production at no-slip walls during vortex-wall collisions
}

Received: 18 May 2009 / Accepted: 20 May 2010 / Published online: 3 July 2010

(C) The Author(s) 2010. This article is published with open access at Springerlink.com

\begin{abstract}
Recently, numerical studies revealed two different scaling regimes of the peak enstrophy $Z$ and palinstrophy $P$ during the collision of a dipole with a no-slip wall [Clercx and van Heijst, Phys. Rev. E 65, 066305, 2002]: $Z \propto \operatorname{Re}^{0.8}$ and $P \propto \operatorname{Re}^{2.25}$ for $5 \times 10^{2} \leq \operatorname{Re} \leq 2 \times 10^{4}$ and $Z \propto \operatorname{Re}^{0.5}$ and $P \propto \operatorname{Re}^{1.5}$ for $\operatorname{Re} \geq 2 \times 10^{4}$ (with Re based on the velocity and size of the dipole). A critical Reynolds number $\operatorname{Re}_{c}$ (here, $\operatorname{Re}_{c} \approx 2 \times 10^{4}$ ) is identified below which the interaction time of the dipole with the boundary layer depends on the kinematic viscosity $\nu$. The oscillating plate as a boundary-layer problem can then be used to mimick the vortex-wall interaction and the following scaling relations are obtained: $Z \propto \operatorname{Re}^{3 / 4}, P \propto \mathrm{Re}^{9 / 4}$, and $\mathrm{d} P / \mathrm{d} t \propto \operatorname{Re}^{11 / 4}$ in agreement with the numerically obtained scaling laws. For $\operatorname{Re} \geq \operatorname{Re}_{c}$ the interaction time of the dipole with the boundary layer becomes independent of the kinematic viscosity and, applying flat-plate boundary-layer theory, this yields: $Z \propto \operatorname{Re}^{1 / 2}$ and $P \propto \operatorname{Re}^{3 / 2}$.
\end{abstract}

Keywords Dipole-wall collision · Confined 2D turbulence $\cdot$ Boundary layers · Vorticity production

\section{Introduction}

A very characteristic process in both decaying and forced two-dimensional (2D) turbulence is the spontaneous development of coherent vortices. In case of a bounded domain with lateral no-slip walls these vortices can collide with the solid boundaries. During such violent events high-amplitude vorticity filaments are generated and subsequently injected into the bulk of the flow. An example is presented in Fig. 1 where a snapshot of 2D decaying turbulence (with initial integral-scale Reynolds number $\mathrm{Re} \approx 10^{5}$ ) in a square domain with no-slip walls is shown. The turbulent flow is clearly dominated by coherent vortices. Many of the small vortices originate from vorticity contained in thin boundary layers, which are generated near the no-slip walls during vortex-wall collisions (left panel). The vorticity production at the no-slip walls is illustrated by viewing a small part of the flow domain near the boundary (right panel). Furthermore, the forces that are exerted on the domain boundaries during individual vortex-wall collisions will provide a net torque on the sidewalls of the container, which might result in a growth of the angular momentum of the flow and possibly to spontaneous spin-up of the flow $[1,2]$.

In this article flows in a confined domain $\Omega$ with no-slip boundaries at $\partial \Omega$ are considered. The $2 \mathrm{D}$ velocity field is denoted by $\mathbf{u}=(u, v)$, and a Cartesian coordinate system is adopted with coordinates $(x, y)$. The total

Communicated by P. R. Spalart

G. H. Keetels · W. Kramer · H. J. H. Clercx · G. J. F. van Heijst

Department of Physics, Eindhoven University of Technology, PO Box 513, 5600 MB, Eindhoven, The Netherlands

H. J. H. Clercx $(\bowtie)$

Department of Applied Mathematics, University of Twente, PO Box 217, 7500 AE, Enschede, The Netherlands

E-mail: h.j.h.clercx@tue.nl 


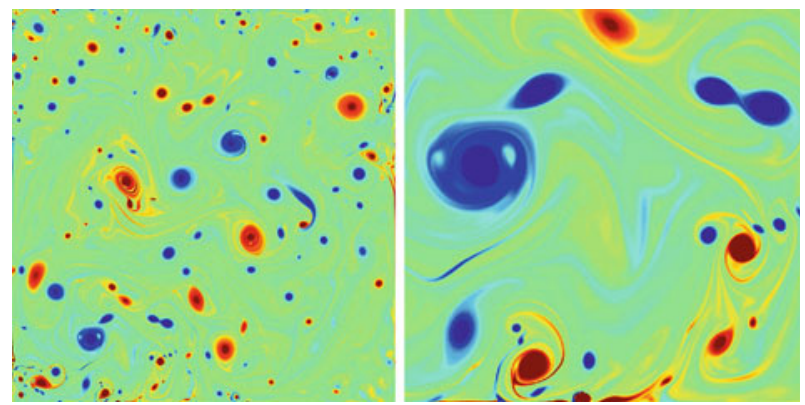

Fig. 1 A vorticity snapshot of a simulation of decaying 2D turbulence on a square domain with no-slip sidewalls with initial integral-scale Reynolds number $\operatorname{Re} \approx 10^{5}$. The left panel shows the full domain with the many coherent vortices. The right panel shows the flow near the left-bottom corner of the domain including evidence of vorticity generation near the no-slip boundary

kinetic energy per unit density is defined as $E(t)=\frac{1}{2} \int_{\Omega}|\mathbf{u}(\mathbf{r}, t)|^{2} \mathrm{~d} A$, with $\mathbf{r}=(x, y)$ and $\mathrm{d} A=\mathrm{d} x \mathrm{~d} y$ an infinitesimal area element of $\Omega$. The evolution equation for $E(t)$ for flows without external forcing reads

$$
\frac{\mathrm{d} E(t)}{\mathrm{d} t}=-2 v Z(t)
$$

Here, $Z(t)$ denotes the total enstrophy of the flow, $Z(t)=\frac{1}{2} \int_{\Omega} \omega^{2}(\mathbf{r}, t) d A$, with $\omega=\frac{\partial v}{\partial x}-\frac{\partial u}{\partial y}$ the vorticity. By considering the Navier-Stokes equations in velocity-vorticity formulation it can be derived that the enstrophy evolves according to

$$
\frac{\mathrm{d} Z(t)}{\mathrm{d} t}=v \oint_{\partial \Omega} \omega(\mathbf{n} \cdot \nabla) \omega \mathrm{d} s-2 v P(t),
$$

where $\mathrm{d} s$ denotes an infinitesimal boundary element of $\partial \Omega$, and $P(t)$ denotes the total palinstrophy of the flow defined as $P=\frac{1}{2} \int_{\Omega}|\nabla \omega(\mathbf{r}, t)|^{2} \mathrm{~d} A$. The first term in (2) demonstrates that enstrophy can be produced at no-slip boundaries. This is a crucial difference compared with flow in a square periodic box, where this integral is essentially zero. The other term represents the dissipation of enstrophy and is present in the case of periodic boundaries as well.

Considering the important role of vortices in bounded turbulence, it is helpful to address the interaction of vortices with solid no-slip boundaries in some detail. In particular, the scaling of the vorticity production with the Reynolds number is of special interest because the boundaries act as vorticity sources (note that vorticity sources are absent in decaying 2D turbulence in periodic domains). It determines the scaling with the Reynolds number of the integral quantities like $Z$ and $P$. In previous studies [3] it has been shown by numerical simulations that the dipole-wall collision is characterized by two scaling regimes. For a Reynolds number $\left(\operatorname{Re}=\frac{U L}{v}\right.$, based on the velocity $U$ and the size $L$ of the dipole, and the kinematic viscosity $v$ of the fluid) larger than approximately $2 \times 10^{4}$ the scaling of vorticity production at the no-slip wall can quite surprisingly be predicted on basis of flat-plate boundary layer theory: $\left.\omega\right|_{\partial \Omega} \propto \operatorname{Re}^{0.5}$. However, for $10^{3} \leq \operatorname{Re} \leq 2 \times 10^{4}$, a different scaling regime is found. In this article the origin of this particular scaling regime is discussed and a model proposed that predicts fairly well the scaling of the integral quantities. It is anticipated that the scaling relations of integral quantities that are obtained for vortex-wall problems can be extended towards fully developed 2D turbulence.

The article is organized as follows: in Sect. 2 the mechanism of vorticity production at no-slip boundaries is briefly reviewed. Subsequently, we focus in Sect. 3 on the dipole-wall collision and recall the previously proposed scaling relations for large Reynolds numbers. Finally, an alternative scaling relation is proposed and validated in Sect. 4. We end this article with a brief conclusion.

\section{Vorticity production at no-slip walls}

To reveal the mechanism of vorticity production at a no-slip wall, Morton [4] has studied the exact solutions of various Stokes problems. A similar approach will be developed in this article. First, the exact solutions of two viscous diffusion problems are briefly reviewed that may serve as analogies for more complicated flow-wall 
interaction problems. Heuristic scaling arguments can be extracted from the viscous solutions, which may be helpful to clarify some scaling issues on the energy, enstrophy, palinstrophy, and the corresponding derivatives in $2 \mathrm{D}$ turbulence confined by no-slip sidewalls.

First we consider vorticity production in the upper half plane above a horizontal flat plate. From the $x$-component of the Navier-Stokes equations in velocity-pressure form, an expression can be derived for the flux of vorticity into the upper half-plane,

$$
-v \frac{\partial \omega}{\partial y}=\frac{1}{\rho} \frac{\partial p}{\partial x}+\frac{\mathrm{d} \tilde{u}}{\mathrm{~d} t} \quad \text { for } \quad y=0, t \in[0, \infty),
$$

where $\tilde{u}$ denotes the velocity of the plate, $p$ is the pressure and $\rho$ represents the fluid density. Equation 3 shows that the flux of enstrophy into the upper half-plane is generated by a pressure gradient $\frac{\partial p}{\partial x}$ along the plate and the acceleration of the plate, given by $\frac{\mathrm{d} \tilde{u}}{\mathrm{~d} t}$. Since the pressure gradient along the boundary and the acceleration of the plate have the same effect on the vorticity production a simple problem will be examined. Consider an oscillating plate that generates a horizontal velocity profile $u(y, t)$ on the upper half-plane $y \geq 0$. The amplitude of the oscillating plate is denoted by $V$ and the frequency by $\omega_{\mathrm{p}}=2 \pi / T_{\mathrm{p}}$ with $T_{\mathrm{p}}$ the period of the oscillation. Only the diffusive part of the $x$-component of the Navier-Stokes equations in velocity-pressure form will be considered. It is assumed that there are no pressure differences in the horizontal direction, thus $\frac{\partial p}{\partial x}=0$. This yields a diffusion problem with the boundary condition $u=V \cos \left(\omega_{\mathrm{p}} t\right)$ at $y=0$. It is further assumed that for $y \rightarrow \infty$ the velocity tends to zero, $u \rightarrow 0$. The solution is $\frac{u}{V}=\exp \left(-y / \delta_{\mathrm{p}}\right) \cos \left(\omega_{\mathrm{p}} t-\frac{y}{\delta_{\mathrm{p}}}\right)$, where $\delta_{\mathrm{p}}=\sqrt{2 v / \omega_{\mathrm{p}}} \propto \sqrt{T_{\mathrm{p}} v}$ represents the Stokes boundary-layer thickness. In the scaling analysis below only the Stokes boundary-layer thickness $\delta_{\mathrm{p}}$ is used, without exploiting the actual solution of the Stokes problem.

For the oscillating plate the vorticity amplitude on the boundary is larger for high frequencies (smaller $T_{\mathrm{p}}$ ), as there is less time available for inward viscous diffusion to reduce the vorticity at the wall. Straightforward calculation yields the following expressions for the vorticity (gradient) at the boundary, the integral quantities $Z$ and $P$, and the time derivatives of the energy and enstrophy, respectively,

$$
\begin{aligned}
& \left.\omega\right|_{\partial \Omega} \propto \frac{V}{\delta_{\mathrm{p}}} \propto \frac{V}{T_{\mathrm{p}}^{1 / 2} \nu^{1 / 2}},\left.\quad \partial_{\mathbf{n}} \omega\right|_{\partial \Omega} \propto \frac{V}{\delta_{\mathrm{p}}^{2}} \propto \frac{V}{T_{\mathrm{p}} v} ; \\
& Z \propto \frac{\mathcal{L} V^{2}}{\delta_{\mathrm{p}}} \propto \frac{\mathcal{L} V^{2}}{T_{\mathrm{p}}^{1 / 2} \nu^{1 / 2}}, \quad P \propto \frac{\mathcal{L} V^{2}}{\delta_{\mathrm{p}}^{3}} \propto \frac{\mathcal{L} V^{2}}{T_{\mathrm{p}}^{3 / 2} \nu^{3 / 2}} ; \\
& \frac{\mathrm{d} E}{\mathrm{~d} t} \propto \mathcal{L} \omega_{\mathrm{p}} V^{2} \delta_{\mathrm{p}} \propto \frac{\mathcal{L} V^{2} v^{1 / 2}}{T_{\mathrm{p}}^{1 / 2}}, \quad \frac{\mathrm{d} Z}{\mathrm{~d} t} \propto \frac{\mathcal{L} \omega_{\mathrm{p}} V^{2}}{\delta_{\mathrm{p}}} \propto \frac{\mathcal{L} V^{2}}{T_{\mathrm{p}}^{3 / 2} \nu^{1 / 2}} .
\end{aligned}
$$

The integrations of the integral quantities $Z$ and $P$ are performed on a rectangular section with a horizontal width $\mathcal{L}$ and depth $\delta_{\mathrm{p}}$ on the upper half-plane. This area $\mathcal{L} \delta_{\mathrm{p}}$ contains almost all vorticity produced at the (oscillating) no-slip wall. In the following, the typical length scale $\mathcal{L}$ will be proportional to the diameter $D$ of the vortex core that collides with the boundary.

In order to verify if a similar vorticity profile develops in a bounded geometry, with consequent scaling behaviour of the integral quantities, the oscillating plate problem is translated to the unit circle $\mathcal{C}$. The solution of the diffusion equation on the unit circle shows very similar behaviour as that of the flat oscillating plate (provided the Stokes boundary-layer thickness is much smaller than the radius of the disk, which in this particular case reads $\left.\delta_{\mathrm{p}} \ll 1\right)$. In this case, the boundary condition for the azimuthal velocity $u_{\phi}$ for $r=1$ is given by $u_{\phi}=V_{\phi} \cos \left(\omega_{\mathrm{p}} t\right)$ for $t \in[0, \infty)$, where $V_{\phi}$ represents the amplitude of the azimuthal velocity at the boundary. It is possible to derive the same scaling relations as assembled in Eqs. 4-6, which signifies that the boundedness of the flow does not affect the validity of the proposed scaling argument.

\section{The dipole-wall collision}

Several studies have been performed on the evolution of a boundary layer at a no-slip wall affected by a single vortex or a few vortices. Peridier et al. [5,6] studied the boundary-layer processes for a vortex above a no-slip wall in a stagnant fluid. Recently, Obabko and Cassel [7] considered a thick-core vortex, which is essentially 
one half of a Lamb dipole. It was assumed that the self-induced velocity balances the free-stream velocity such that the vortex remains at a fixed location with respect to the wall. The presence of the vortex induces an adverse pressure gradient along the boundary which can be related to a flux of vorticity into the upper plane, see Eq. 3. This results in the formation of a recirculation zone followed by a detachment of the boundary layer.

In decaying $2 \mathrm{D}$ turbulence a vortex is in general not fixed at a certain position in the domain. Due to the induced velocity from the other vortices a vortex has a net drift velocity and can consequently collide with the domain boundaries. This process can be mimicked by a dipole translating towards a no-slip wall. The first numerical study of a dipolar vortex colliding with a no-slip wall has been performed by Orlandi [8]. A decade later Clercx and van Heijst [3] performed a numerical dipole-wall collision study at extremely high Reynolds numbers in order to quantify the Reynolds number dependence of the enstrophy and palinstrophy production at the instant of collision. Recently, Kramer et al. [9] reported about a detailed analysis of the dipole-wall collision problem. In the latter study many of the small-scale vorticity features reported by Obabko and Cassel [7] have been observed as well.

In their numerical study, Clercx and van Heijst [3] recognized two different scaling regimes of the peak enstrophy and palinstrophy during the first collision of the dipole with the no-slip boundaries. For relatively low Reynolds number they observed

$$
Z \propto \operatorname{Re}^{0.8}, \quad P \propto \operatorname{Re}^{2.25} \quad \text { for } 5 \times 10^{2} \leq \mathrm{Re} \leq 2 \times 10^{4},
$$

and for higher Reynolds numbers it was found that

$$
Z \propto \operatorname{Re}^{0.5}, \quad P \propto \operatorname{Re}^{1.5} \quad \text { for } \quad \operatorname{Re} \geq 2 \times 10^{4} .
$$

The results (7) and (8) imply that the decay of energy is proportional to $\mathrm{Re}^{-0.2}$ for the lower Reynolds numbers and becomes proportional to $\mathrm{Re}^{-0.5}$ in the large Reynolds number limit, see Eq. 1 . Signs of the presence of the first regime have also been found for decaying 2D turbulence simulations, see Ref. [10]. This decay scenario is markedly different compared with the periodic case where the energy decay is proportional to $\mathrm{Re}^{-1}$.

Using steady boundary-layer theory Clercx and van Heijst [3] could confirm the scaling results (8). They also verified that the circulation of the boundary layer is virtually independent of the Reynolds number for $\operatorname{Re} \geq 10^{4}$. Furthermore, for the lower Reynolds numbers some dependence of the circulation on the Reynolds number was found, which might be responsible for the slight increase of the theoretical scaling exponents. Indeed, some dependence of the circulation on the Reynolds number could justify a small increase of the scaling exponents of the enstrophy and palinstrophy of approximately 0.2 . This might explain the increase of the exponent that corresponds with the total enstrophy from the theoretical value of 0.5 to 0.7 . However, it strongly underestimates the observed scaling exponent for the palinstrophy with a theoretical value of 1.5 to 1.7, whereas the measured exponent is 2.25. Apparently, an alternative explanation for the observed scaling exponents is needed.

\section{Alternative scaling relations for boundary-layer vorticity}

Two typical time-scales can be associated with the dipole-wall collision problem. The first time scale, $T_{\mathrm{a}}$, can be related to the vortex-wall approach. It is a measure of the time-span between the instant a boundary layer starts to form and the instant of collision. For sufficiently high Reynolds number it can be assumed that the amount of dissipation of the total kinetic energy of the vortex in the interior of the domain can be neglected. Therefore, the time scale $T_{\mathrm{a}}$ can be estimated by means of the translation speed $V$ and the diameter $D$ of the vortex core,

$$
T_{\mathrm{a}} \propto \frac{D}{V} .
$$

The second time scale is a measure of the duration of the collision or, in other words, the contact time with the viscous boundary layer. Note that as the dipolar vortex approaches the wall, a boundary layer is formed due to the induction of velocity in the near-wall region. As the primary vortex cores move back into the interior of the flow domain the magnitude of the velocity in the boundary layer will decrease again. A typical shape of the vortex trajectory during the collision with the wall is given in Fig. 2. The part of the trajectory, from the moment the nose of the dipole hits the kinetic boundary layer for the first time till the enstrophy generated near the wall is maximum, represents the first part of the collision. It is indicated by the thick gray segment of the 


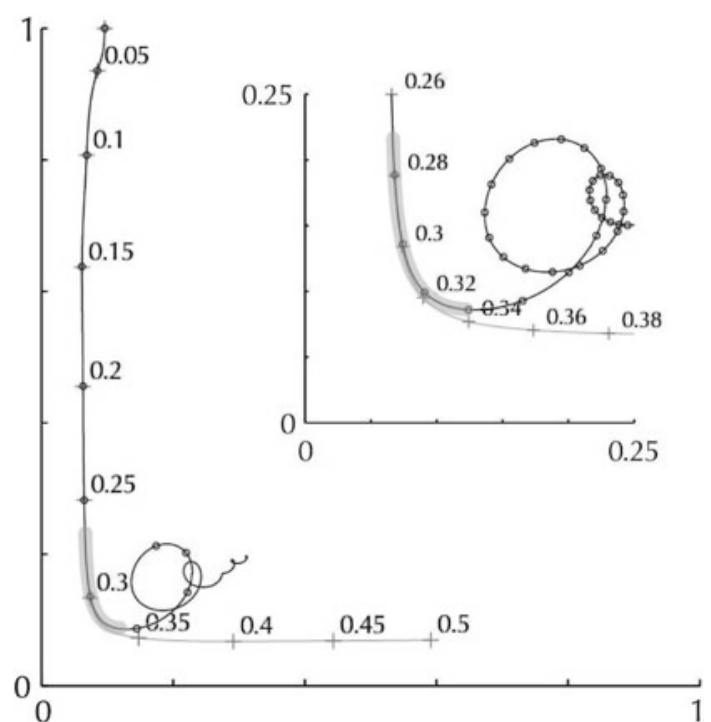

Fig. 2 Trajectory of a dipole approaching a stress-free or a no-slip wall for $\mathrm{Re}=1250$. The normal dipole-wall collision is symmetric with respect to the wall-normal direction ( $y$-axis) and it suffices to show the trajectory of one vortex core only. The path of the dipole colliding with a no-slip wall (black) is compared with the path in case a stress-free boundary condition is applied (gray). The position of the maximum core vorticity at specific equidistant times is indicated by the symbols (plusses and circles for the stress-free and no-slip case, respectively). These specific times are denoted by the labels (only the first five for the no-slip case are given). The part of the path indicating the duration of the first enstrophy increase is indicated by the thick gray segment of the trajectory. This period starts when the dipole first hits the boundary layer and ends when the dipole core is closest to the wall and represent $T_{\mathrm{p}} / 4$, with $T_{\mathrm{p}}$ the duration of the collision, see Eq. 10. Figure is adopted from Kramer et al. [9].

trajectory shown in Fig. 2. The duration is found to be equivalent to a quarter of the collision period $T_{\mathrm{p}}$ defined below, see Eq. 10. It does not require much imagination to realize that the vortex rebound can be compared with half a period of the oscillating plate problem considered in Sect. 2: now it is the fluid that exhibits oscillatory motion, whereas the boundary is fixed. Note that the vorticity is produced due to the presence of a pressure gradient along the wall, which is equivalent to an acceleration of the wall, as can be seen in the vorticity flux Eq. 3.

If we adopt the oscillating plate as an analogous boundary-layer problem, the scaling relations assembled in Eqs. 4-6 can be used to predict the scaling of the enstrophy and palinstrophy in the viscous boundary layer of the dipole-wall problem.

We want to express the scaling relations in terms of the Reynolds number. Therefore, it is essential to express the second time scale, now denoted by $T_{\mathrm{p}}$, in terms of $D, V$ and $\nu$. During the collision, the wallnormal component of the momentum of the dipole is destroyed, hence $T_{\mathrm{p}} \propto \rho D^{2} v_{\perp} / F$. Here, $v_{\perp}$ is the normal-wall component of the velocity of the vortex core at the instant of collision with the top of the boundary layer. A scaling relation for the force $F$ can be derived from the work required to depress the boundary layer, thus $F \propto \rho D^{2} v_{\perp}^{2} / \delta$. This results in $T_{\mathrm{p}} \propto \delta / v_{\perp}$. For low Reynolds numbers, $\operatorname{Re}<\operatorname{Re}_{c}$, the boundary-layer thickness can be expressed as $\delta \propto \sqrt{v T_{\mathrm{a}}}$ and the duration of the collision scales like

$$
T_{\mathrm{p}} \propto \frac{\sqrt{v T_{\mathrm{a}}}}{v_{\perp}} \propto \frac{\sqrt{v D}}{V^{3 / 2}} \quad \text { for } \operatorname{Re}<\operatorname{Re}_{c} .
$$

Note that $v_{\perp}$ is smaller than the translation speed of the vortex $V$, because the trajectory strongly bends during the vortex-wall approach. We consider now a sufficiently high Reynolds number, typically $\operatorname{Re} \geq 1000$. During the first part of the collision the vortex cores follow the same trajectory as in the stress-free case, which is virtually $\mathrm{Re}$-independent for $\mathrm{Re} \gtrsim 1000$. In the range $1000 \leq \mathrm{Re} \leq 20000$ the approach time decreases about $10 \%$. Hence, the velocity component $v_{\perp}$ does not depend significantly on the Reynolds number in this regime. On the other hand, the viscous boundary layer is thicker for lower Reynolds numbers or larger values of the kinematic viscosity. This will result in a longer duration of the collision, as the boundary layer will resist the motion of the incoming vortex over a larger distance. Realize that the leading-order pressure is constant vertically across the boundary layer, which can readily be derived from the wall-normal component of the momentum equation at the no-slip boundary. 
Inserting the expression (10) for $T_{\mathrm{p}}$ in the range $\mathrm{Re}<\mathrm{Re}_{c}$ into the scaling expression for the integral quantities of the oscillating plate problem, see Eq. 5 where we have now used $\mathcal{L}=D$, yields $Z \propto \operatorname{Re}^{3 / 4}$ and $P \propto \operatorname{Re}^{9 / 4}$. These scaling relations agree reasonably well with the scaling relation (7) observed in the numerical study on dipole-wall collisions conducted by Clercx and van Heijst [3] with $\operatorname{Re} \lesssim \operatorname{Re}_{c} \approx 20000$.

With increasing Reynolds number the boundary-layer thickness decreases according to $\delta_{\mathrm{p}} \propto 1 / \sqrt{\mathrm{Re}}$. Above $\operatorname{Re}_{c}$ the boundary layer has become so thin that it is hardly deformed by the approaching dipole. Moreover, the circulation in the boundary layer becomes independent of the Reynolds number [3]. The destruction of the wall-normal component of momentum of the dipole is assumed to depend only on $D$ and $V$, the effect of the kinematic viscosity being negligibe (as in the free-slip case). The collision time scale is then defined by $V$ and $D$,

$$
T_{\mathrm{p}} \propto \frac{D}{V} \quad \text { for } \operatorname{Re}>\operatorname{Re}_{c} .
$$

The results obtained by applying flat-plate boundary-layer theory should be recovered. For the high Reynolds number regime $\mathrm{Re}>\operatorname{Re}_{c}$ it is now found, by inserting (11) into Eq. 5, that $Z \propto \operatorname{Re}^{1 / 2}$ and $P \propto \operatorname{Re}^{3 / 2}$, consistent with Ref. [3] for $\mathrm{Re} \geq 20000$.

Although the scaling result shows satisfactory correspondence with the numerical data for enstrophy and palinstrophy, it is important to verify if it is indeed conjecture (10) that explains the anomalous scaling behaviour for $\operatorname{Re} \leq 20000$. It is difficult, however, to precisely obtain the value of the time scale $T_{\mathrm{p}}$. It can be based, for instance, on the width of the enstrophy or palinstrophy peaks during the collision. In the present study we used the following expression to compute a quantitative value for the collision time: $T_{\mathrm{p}}=2 \pi P_{\max } /\left(\frac{\mathrm{d} P}{\mathrm{~d} t}\right)_{\max }$, with $P_{\max }$ the maximum value of the palinstrophy and $\left(\frac{\mathrm{d} P}{\mathrm{~d} t}\right)_{\max }$ the steepest part of the time rate of change of the palinstrophy before the collision of the dipole with the wall. The curves obtain a more spiky appearance for high Reynolds numbers, which could indicate a decreasing duration of the dipole-wall collision. A convenient method to verify the validity of the scaling (10) is to consider the time derivative of the palinstrophy $P(t)$. Following the same oscillating plate analogy an expression can be derived for the time derivative of the palinstrophy which is proportional to

$$
\frac{\mathrm{d} P}{\mathrm{~d} t} \propto \frac{D V^{2}}{T_{\mathrm{p}}^{5 / 2} v^{3 / 2}} .
$$

The conjecture for the scaling of the collision time (10) yields then an estimate for the time derivative of the palinstrophy according to $\frac{\mathrm{d} P}{\mathrm{~d} t} \propto \mathrm{Re}^{11 / 4}$. Figure 3 assembles the data of the enstrophy, palinstrophy, and the time derivative of the palinstrophy for different Reynolds numbers in a double logarithmic plot. It can be seen that for $1000<\operatorname{Re}<20000$ all the estimates are in excellent agreement with the alternative scaling proposal. Small deviations can be observed for the data obtained with $\operatorname{Re} \leq 1250$.

Note that it is assumed that the typical velocity $V$ is constant for $500<\operatorname{Re}<2 \times 10^{4}$. The rms velocity at the instant of the collision changes significantly between Reynolds numbers of 625 and 2500, namely from 0.79 to 0.93 , respectively. For higher Reynolds numbers the difference is, however, negligible since the rms
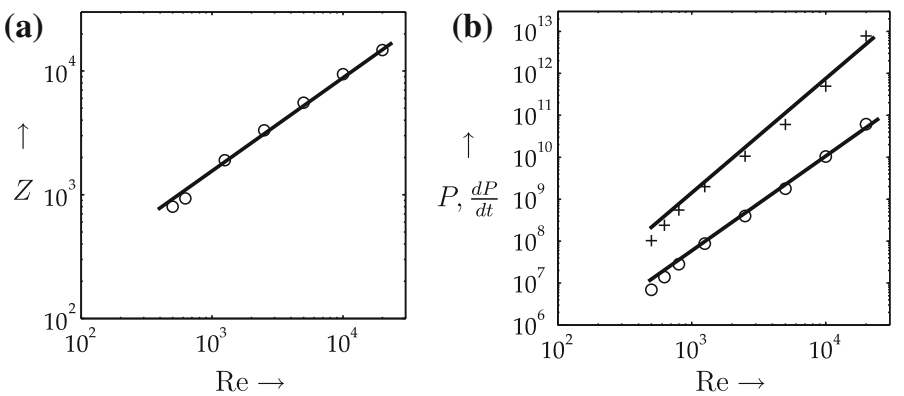

Fig. 3 Maxima of a the enstrophy $Z$ and $\mathbf{b}$ the palinstrophy $P$ (open circles) and the maximum of $\mathrm{d} P / \mathrm{d} t$ (plus signs). Reference line $\operatorname{Re}^{0.75}$ for $Z$ in (a). Reference lines $\operatorname{Re}^{2.25}$ and $\operatorname{Re}^{2.75}$ for $P$ and $\mathrm{d} P / \mathrm{d} t$, respectively, in (b). 
velocity is 0.96 for $\mathrm{Re}=20000$, thus within a few procent error margin of the corresponding value for the $\operatorname{Re}=2500$ case. Recall that in Kramer et al. [9] it was observed that the shape of the vortex for $\operatorname{Re} \leq 1250$ depends on the Reynolds number. Furthermore, the approach of the dipole has a different appearance due to interaction with the detached vorticity layers for the lower Reynolds number cases. These effects might explain the minor deviations observed for Re $\leq 1250$ in Fig. 3 .

It is also possible to obtain an estimate for the pressure at the boundary by the vorticity flux Eq. 3 and the estimate in the oscillating plate problem for the velocity gradients perpendicular to the wall, yielding

$$
\left.p\right|_{\partial \Omega} \propto \frac{\rho D V}{T_{\mathrm{p}}} .
$$

Note that for an incompressible flow only the pressure difference is relevant. Here we set the domain averaged pressure equal to zero. Equation 13 reveals an intimate relationship between the collision time $T_{\mathrm{p}}$ and the pressure in the boundary layer. Therefore, the usual assumption that the pressure becomes finite in the limit of vanishing viscosity is consistent with the conjecture (11) of a finite value of the collision time $T_{\mathrm{p}}$. The impulse exerted by the boundary layers on the colliding vortex can be estimated by using the estimate (13) for the pressure and the collision time $T_{\mathrm{p}}, I \propto \rho D^{2} V$. The right-hand side is proportional to the change of momentum as the dipole collides with the boundary layer. Note that for increasing Reynolds number in the range $\operatorname{Re}<\operatorname{Re}_{c}$ the collision time $T_{\mathrm{p}}$ decreases according to (10), and the pressure increases with $1 / T_{\mathrm{p}}$ such that the net change of momentum is essentially constant.

The current scaling analysis is conducted for the normal dipole-wall collision experiment. In the numerical study by Clercx and van Heijst [3] a second scenario has been considered: the oblique dipole-wall collision. Here, the initial trajectory of the dipole had an angle of $\alpha=60^{\circ}$ with respect to the no-slip wall (instead of $\alpha=90^{\circ}$ for the normal collision). The newly derived scaling laws turn out to fit extremely well with this dataset too, see Ref. [3]. It also explains the somewhat smaller values of $Z$ and $P$ for the oblique collision experiment. The normal velocity component in Eqs. 10 and 11 has the following form: $v_{\perp} \propto V \sin \alpha$. For $\operatorname{Re}<\operatorname{Re}_{c}$ this yields the same scaling with Re as before but with reduced values for the enstrophy and palinstrophy according to $Z \propto(\sin \alpha)^{11 / 4}$ and $P \propto(\sin \alpha)^{17 / 4}$. The reduction in enstrophy and palinstrophy should then be 67 and $54 \%$, respectively. The data from Ref. [3] show a reduction of approximately $70 \pm 5 \%$ and $60 \pm 5 \%$ for the enstrophy and palinstrophy, respectively, in very good agreement with the predictions. Similar conclusions can be drawn for the range $\operatorname{Re}>\operatorname{Re}_{c}$.

\section{Conclusions}

The two different scaling regimes of the peak enstrophy $Z$ and palinstrophy $P$ during the collision of a dipole with a no-slip wall, as observed numerically by Clercx and van Heijst [3], have been retrieved theoretically. A critical Reynolds number $\operatorname{Re}_{c}$ (here $\operatorname{Re}_{c} \approx 2 \times 10^{4}$ ) is identified below which the interaction time of the dipole with the boundary layer depends on the kinematic viscosity $v$. The oscillating plate as a boundary-layer problem can then be used to obtain the following scaling relations: $Z \propto \operatorname{Re}^{3 / 4}, P \propto \operatorname{Re}^{9 / 4}$, and $\mathrm{d} P / \mathrm{d} t \propto \operatorname{Re}^{11 / 4}$, in agreement with the numerically obtained scaling laws. For $\operatorname{Re} \geq \operatorname{Re}_{c}$ the interaction time of the dipole with the boundary layer becomes independent of the kinematic viscosity and, applying flat-plate boundary-layer theory (see Ref. [3]), this yields: $Z \propto \operatorname{Re}^{1 / 2}$ and $P \propto \operatorname{Re}^{3 / 2}$.

It is rather surprising that relatively simple scaling arguments can be applied to the quite complicated collision process of a dipole with a no-slip wall. The study of Kramer et al. [9] reports on the formation of a secondary boundary layer and consequent development of shear layer instabilities as soon as the dipolar vortex collides with the wall for Reynolds numbers larger than 5000. Therefore, it is quite remarkable that a simplified unsteady boundary-layer model can still explain the anomalous scaling of the enstrophy and palinstrophy at the instant of collision with satisfactory agreement between the scaling model and the numerical data.

We expect that both scaling regimes affect forced and decaying 2D turbulence in confined domains (with no-slip sidewalls) as the vorticity production at no-slip walls is substantial. Current numerical studies are limited to integral-scale Reynolds numbers of $\mathcal{O}\left(10^{5}\right)$ implying that the Reynolds number based on the larger vortices (that eventually collide with the boundaries) will be in the range $10^{3}-10^{4}$. In Ref. [10] evidence is presented that indeed the anomalous scaling regime can be observed. It is expected that either of the scaling regimes will be applicable, thus substantially enhancing the dissipation of the flow. It is not yet known which regime eventually dominates the dissipation in bounded 2D turbulence; this requires further large-scale numerical simulations. 
Acknowledgements The authors gratefully acknowledge financial support from the Dutch Foundation for Fundamental Research on Matter (FOM) and from the Technology Foundation (STW) under Grant No. ESF.6239, which are both financially supported by the Nederlandse Organisatie voor Wetenschappelijk Onderzoek (Netherlands Organization for Scientific Research, NWO). Part of this study was sponsored by the Stichting Nationale Computerfaciliteiten (National Computing Facilities Foundation, $\mathrm{NCF}$ ) for the use of supercomputer facilities, with financial support from NWO.

Open Access This article is distributed under the terms of the Creative Commons Attribution Noncommercial License which permits any noncommercial use, distribution, and reproduction in any medium, provided the original author(s) and source are credited.

\section{References}

1. Clercx, H.J.H., Maassen, S.R., van Heijst, G.J.F.: Spontaneous spin-up during the decay of 2D turbulence in a square container with rigid boundaries. Phys. Rev. Lett. 80, 5129-5132 (1998)

2. Clercx, H.J.H., Nielsen, A.H., Torres, D.J., Coutsias, E.A.: Two-dimensional turbulence in square and circular domains with no-slip walls. Eur. J. Mech. B-Fluids 20, 557-576 (2001)

3. Clercx, H.J.H., van Heijst, G.J.F.: Dissipation of kinetic energy in two-dimensional bounded flows. Phys. Rev. E 65(066305), 1-4 (2002)

4. Morton, B.R.: The generation and decay of vorticity. Geophys. Astrophys. Fluid Dyn. 28, 277-308 (1984)

5. Peridier, V.J., Smith, F.T., Walker, J.D.A.: Vortex-induced boundary layer separation. Part 1. The unsteady limit problem $\mathrm{Re} \rightarrow \infty$. J. Fluid. Mech. 232, 99-131 (1991)

6. Peridier, V.J., Smith, F.T., Walker, J.D.A.: Vortex-induced boundary layer separation. Part 2. Unsteady interacting boundary-layer theory. J. Fluid. Mech. 232, 133-165 (1991)

7. Obabko, A.V., Cassel, K.V.: Navier-Stokes solutions of unsteady separation induced by a vortex. J. Fluid Mech. 465, 99-130 (2002)

8. Orlandi, P.: Vortex dipole rebound from a wall. Phys. Fluids A 2, 1429-1436 (1990)

9. Kramer, W., Clercx, H.J.H., van Heijst, G.J.F.: Vorticity dynamics of a dipole colliding with a no slip wall. Phys. Fluids 19(126603), 1-13 (2007)

10. Keetels, G.H.: Fourier spectral computation of geometrically confined two-dimensional flows. PhD Thesis, Eindhoven University of Technology, The Netherlands (2008). 